CRYSTALLOGRAPHIC COMMUNICATIONS

ISSN 2056-9890

Received 16 December 2019

Accepted 8 January 2020

Edited by W. T. A. Harrison, University of Aberdeen, Scotland

Keywords: crystal structure; potassium phosphate; hydrogen bonding; absolute structure; hydrate.

CCDC reference: 1976170

Supporting information: this article has supporting information at journals.iucr.org/e

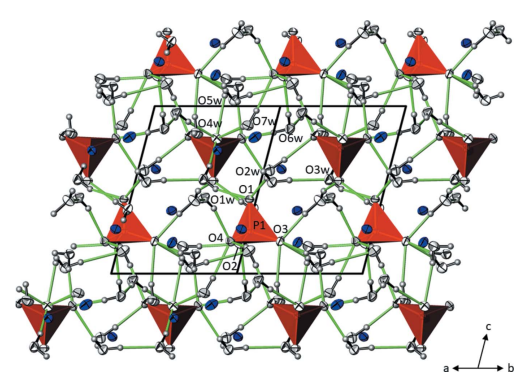

OPEN $\odot$ ACCESS

\section{Crystal structure of the deuterated heptahydrate of potassium phosphate, $\mathrm{K}_{3} \mathrm{PO}_{4} \cdot 7 \mathrm{D}_{2} \mathrm{O}$}

\author{
Matthias Weil ${ }^{a *}$ and Berthold Stöger ${ }^{b}$ \\ anstitute for Chemical Technologies and Analytics, Division of Structural Chemistry, Vienna University of Technology, \\ Getreidemarkt 9/164-SC, A-1060 Vienna, Austria, and 'b-Ray Centre, Getreidemarkt 9, A-1060 Vienna, Austria. \\ *Correspondence e-mail: matthias.weil@tuwien.ac.at
}

Deuterated potassium orthophosphate heptahydrate, $\mathrm{K}_{3} \mathrm{PO}_{4} \cdot 7 \mathrm{D}_{2} \mathrm{O}$, crystallizes in the Sohnke space group $P 2_{1}$, and its absolute structure was determined from 2017 Friedel pairs [Flack parameter $0.004(16)]$. Each of the three crystallographically unique $\mathrm{K}^{+}$cations is surrounded by six water molecules and one oxygen atom from the orthophosphate group, using a threshold for $\mathrm{K}-\mathrm{O}$ bonds of $3.10 \AA$. The highly irregular coordination polyhedra are linked by corner- and edge-sharing into a three-dimensional network that is consolidated by an intricate network of $\mathrm{O}-\mathrm{D}$. . O hydrogen bonds of medium strength.

\section{Chemical context}

Following projects devoted to studying the formation and crystal chemistry of hydrous arsenate and phosphate phases of monovalent metals, viz. $\mathrm{NaH}_{2} \mathrm{AsO}_{4}$ (Ring et al., 2017), $\mathrm{K}_{2} \mathrm{HAsO}_{4}\left(\mathrm{H}_{2} \mathrm{O}\right)_{2.5}$ and $\mathrm{K}_{2} \mathrm{HAsO}_{4}\left(\mathrm{H}_{2} \mathrm{O}\right)_{6}$ (Stöger et al., 2012),

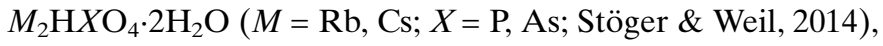
and several acidic thallium(I) arsenate phases (Schroffenegger et al., 2019), we became interested in the system $\mathrm{K}_{3} \mathrm{PO}_{4} / \mathrm{H}_{2} \mathrm{O}$. Although hydrate phases of potassium orthophosphate have been known for a very long time to exist for the 3-hydrate and the 7-hydrate (Gmelin, 1938), crystal-structure determinations of these two phases or of any other hydrate of $\mathrm{K}_{3} \mathrm{PO}_{4}$ have not been reported so far. Previous investigations on the trihydrate revealed that the crystal structure of $\mathrm{K}_{3} \mathrm{PO}_{4} \cdot 3 \mathrm{H}_{2} \mathrm{O}$ is incommensurately modulated below $300 \mathrm{~K}$ (Stöger, 2020). To better elucidate the role of hydrogen bonding in this structure with the aid of single-crystal neutron diffraction, we started crystalgrowth experiments to obtain the deuterium analogue $\mathrm{K}_{3} \mathrm{PO}_{4} \cdot 3 \mathrm{D}_{2} \mathrm{O}$. The title compound, $\mathrm{K}_{3} \mathrm{PO}_{4} \cdot 7 \mathrm{D}_{2} \mathrm{O}$, was the unexpected product of such a crystallization attempt at temperatures below the freezing point of pure water, and its crystal structure is reported here.

\section{Structural commentary}

Taking $3.1 \AA$ as the upper limit of $\mathrm{K}-\mathrm{O}$ bond lengths in the first coordination sphere, each of the three crystallographically independent potassium cations is surrounded by six water molecules and one oxygen atom of the phosphate group (Fig. 1). The highly irregular coordination polyhedra show $\mathrm{K}-\mathrm{O}$ bond lengths ranging between 2.6665 (9) and $3.0151 \AA$ (Table 1). The overall mean of $2.821 \AA$ for the 21 bonds is in good agreement with the value of $2.861 \AA$ calculated from 469 individual $\mathrm{K}-\mathrm{O}$ bonds in crystal structures with coordination 
Table 1

Selected bond lengths $(\AA)$.

\begin{tabular}{llll}
\hline $\mathrm{K} 1-\mathrm{O} 5 w$ & $2.7153(10)$ & $\mathrm{K} 2-\mathrm{O} 6 w^{\mathrm{i}}$ & $3.0151(10)$ \\
$\mathrm{K} 1-\mathrm{O} 1 w$ & $2.7183(11)$ & $\mathrm{K} 3-\mathrm{O} 2 w^{\mathrm{i}}$ & $2.6665(9)$ \\
$\mathrm{K} 1-\mathrm{O} 7 w$ & $2.7381(10)$ & $\mathrm{K} 3-\mathrm{O} 4^{\mathrm{iv}}$ & $2.7867(9)$ \\
$\mathrm{K} 1-\mathrm{O} 6 w$ & $2.7532(9)$ & $\mathrm{K} 3-\mathrm{O} 4 w^{\mathrm{v}}$ & $2.7983(10)$ \\
$\mathrm{K} 1-\mathrm{O} 3 w$ & $2.8479(9)$ & $\mathrm{K} 3-\mathrm{O} 5 w^{\mathrm{vi}}$ & $2.8344(10)$ \\
$\mathrm{K} 1-\mathrm{O} 2 w$ & $2.8486(9)$ & $\mathrm{K} 3-\mathrm{O} 1 w^{\mathrm{iv}}$ & $2.8394(10)$ \\
$\mathrm{K} 1-\mathrm{O} 1$ & $2.9757(9)$ & $\mathrm{K} 3-\mathrm{O} 7 w^{\mathrm{vi}}$ & $2.9094(9)$ \\
$\mathrm{K} 2-\mathrm{O} 1$ & $2.7317(10)$ & $\mathrm{K} 3-\mathrm{O} 5 w$ & $2.9246(10)$ \\
$\mathrm{K} 2-\mathrm{O} 4 w$ & $2.7391(10)$ & $\mathrm{P} 1-\mathrm{O} 1$ & $1.5414(8)$ \\
$\mathrm{K} 2-\mathrm{O} 7 w$ & $2.7659(9)$ & $\mathrm{P} 1-\mathrm{O} 2$ & $1.5440(8)$ \\
$\mathrm{K} 2-\mathrm{O} 1 w^{\mathrm{i}}$ & $2.7836(9)$ & $\mathrm{P} 1-\mathrm{O} 4$ & $1.5472(10)$ \\
$\mathrm{K} 2-\mathrm{O} 2 w^{\mathrm{ii}}$ & $2.8269(9)$ & $\mathrm{P} 1-\mathrm{O} 3$ & $1.5523(8)$ \\
$\mathrm{K} 2-\mathrm{O} 3 w^{\mathrm{iii}}$ & $3.0144(9)$ & &
\end{tabular}

Symmetry codes: (i) $x-1, y, z$; (ii) $-x, y-\frac{1}{2},-z+1$; (iii) $-x, y+\frac{1}{2},-z+1$; (iv) $-x+1, y+\frac{1}{2},-z+1$; (v) $x+1, y, z$; (vi) $-x+1, y+\frac{1}{2},-z+2$.

Table 2

Hydrogen-bond geometry $\left(\AA,^{\circ}\right)$.

\begin{tabular}{|c|c|c|c|c|}
\hline$D-\mathrm{H} \cdots A$ & $D-\mathrm{H}$ & $\mathrm{H} \cdots A$ & $D \cdots A$ & $D-\mathrm{H} \cdots A$ \\
\hline $\mathrm{O} 1 w-\mathrm{D} 11 \cdots \mathrm{O} 3 w^{\mathrm{iv}}$ & $0.86(2)$ & $1.91(2)$ & $2.7255(13)$ & $158(2)$ \\
\hline $\mathrm{O} 1 w-\mathrm{D} 12 \cdots \mathrm{O} 4$ & $0.78(2)$ & $1.98(2)$ & $2.7391(11)$ & $164(2)$ \\
\hline $\mathrm{O} 2 w-\mathrm{D} 21 \cdots \mathrm{O} 2^{\mathrm{iii}}$ & $0.87(2)$ & $1.85(2)$ & $2.7149(13)$ & $177(2)$ \\
\hline $\mathrm{O} 2 w-\mathrm{D} 22 \cdots \mathrm{O} 1$ & $0.73(2)$ & $1.99(2)$ & $2.7029(11)$ & $167(3)$ \\
\hline $\mathrm{O} 3 w-\mathrm{D} 31 \cdots \mathrm{O} 1$ & $0.80(2)$ & $1.97(2)$ & $2.7242(11)$ & $159(2)$ \\
\hline $\mathrm{O} 3 w-\mathrm{D} 32 \cdots \mathrm{O} 3^{\mathrm{ii}}$ & $0.98(3)$ & $1.77(3)$ & $2.7395(14)$ & $171(2)$ \\
\hline $\mathrm{O} 4 w-\mathrm{D} 41 \cdots \mathrm{O} 3^{\mathrm{vii}}$ & $0.80(2)$ & $2.10(2)$ & $2.8870(14)$ & $169(2)$ \\
\hline $\mathrm{O} 4 w-\mathrm{D} 42 \cdots \mathrm{O} 2^{\mathrm{iii}}$ & $0.79(2)$ & $1.97(2)$ & $2.7679(13)$ & $177(2)$ \\
\hline $\mathrm{O} 5 w-\mathrm{D} 51 \cdots \mathrm{O} 6 w^{\mathrm{vi}}$ & $0.80(2)$ & $2.11(2)$ & $2.9025(13)$ & $168(2)$ \\
\hline $\mathrm{O} 5 w-\mathrm{D} 52 \cdots \mathrm{O} 3^{\mathrm{viii}}$ & $0.80(2)$ & $1.92(2)$ & $2.6944(12)$ & $166(2)$ \\
\hline $\mathrm{O} 6 w-\mathrm{D} 61 \cdots \mathrm{O} 2^{\mathrm{viii}}$ & $0.75(2)$ & $1.96(2)$ & $2.7087(12)$ & $176(2)$ \\
\hline $\mathrm{O} 6 w-\mathrm{D} 62 \cdots \mathrm{O} 4^{\mathrm{ix}}$ & $0.85(2)$ & $1.86(2)$ & $2.6931(12)$ & $165(2)$ \\
\hline $\mathrm{O} 7 w-\mathrm{D} 71 \cdots \mathrm{O} 3^{\mathrm{ii}}$ & $0.78(2)$ & $2.02(2)$ & $2.7498(12)$ & $155(2)$ \\
\hline $\mathrm{O} 7 w-\mathrm{D} 72 \cdots \mathrm{O} 4^{\mathrm{vii}}$ & $0.82(2)$ & $1.97(2)$ & $2.7859(13)$ & $171(2)$ \\
\hline
\end{tabular}

Symmetry codes: (ii) $\quad-x, y-\frac{1}{2},-z+1$; (iii) $\quad-x, y+\frac{1}{2},-z+1$; $\quad$ (iv) $-x+1, y+\frac{1}{2},-z+1$; (vi) $-x+1, y+\frac{1}{2},-z+2$; (vii) $x, y, z+1$; (viii) $x+1, y, z+1$; (ix) $-x+1, y-\frac{1}{2},-z+1$.

numbers of 7 for the potassium cation (Gagné \& Hawthorne, 2016). The $\left[\mathrm{K}\left(\mathrm{D}_{2} \mathrm{O}\right)_{6} \mathrm{O}\right]$ polyhedra share corners and edges to build up a three-dimensional network (Fig. 2). Each water molecule is a donor group of two slightly bent $\mathrm{O}-\mathrm{D} \cdots \mathrm{O}$ hydrogen bonds, but only two of the water molecules $(\mathrm{O} 3 w$,

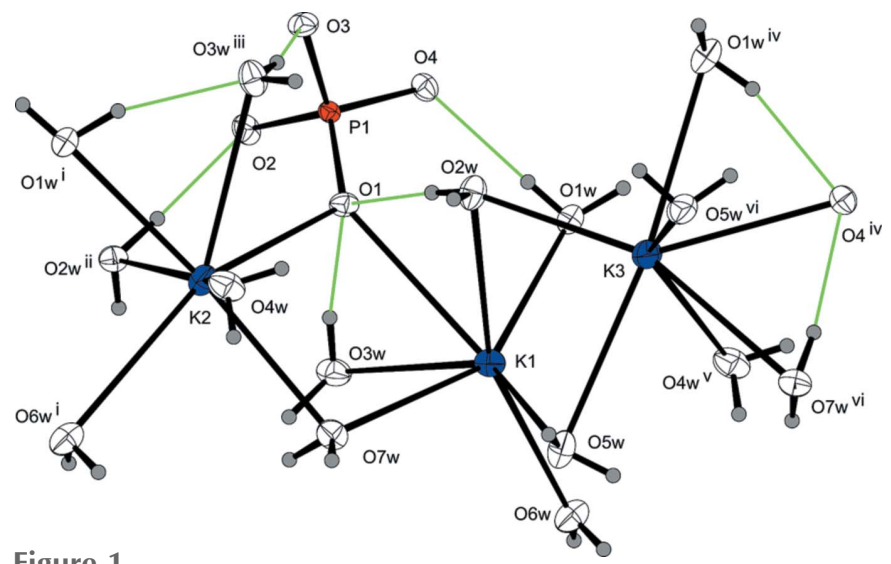

Figure 1

The expanded asymmetric unit of $\mathrm{K}_{3} \mathrm{PO}_{4} \cdot 7 \mathrm{D}_{2} \mathrm{O}$ showing the complete potassium coordination polyhedra. Displacement ellipsoids are displayed at the $74 \%$ probability level; $\mathrm{O}-\mathrm{D} \cdots \mathrm{O}$ hydrogen bonds are indicated by green lines; symmetry codes refer to Table 1.

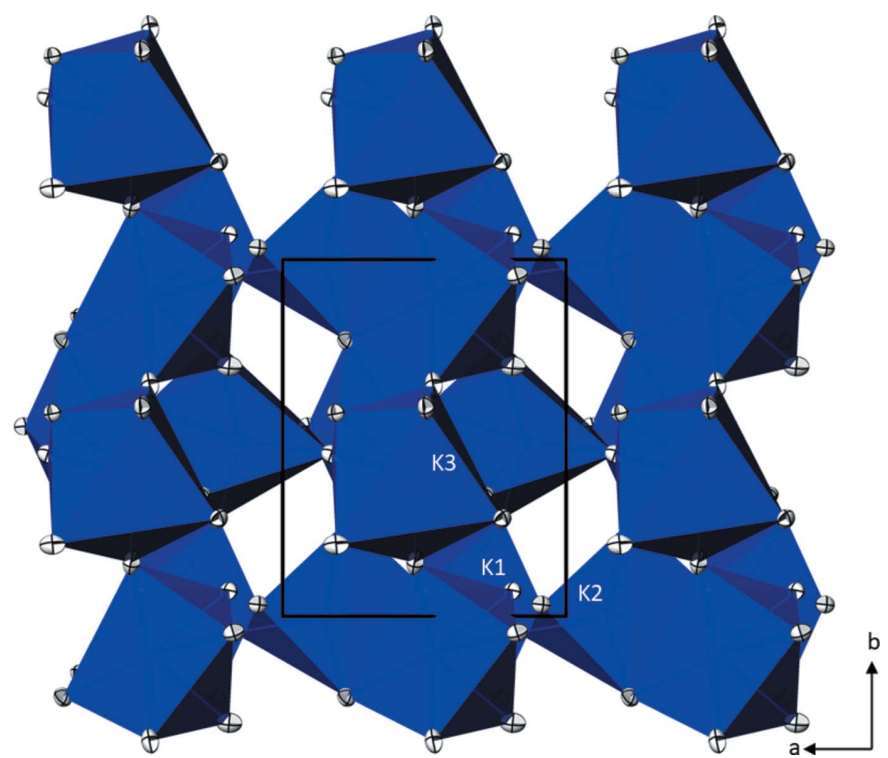

Figure 2

Network of corner- and edge-sharing $\left[\mathrm{KO}_{7}\right]$ polyhedra in the crystal structure of $\mathrm{K}_{3} \mathrm{PO}_{4} \cdot 7 \mathrm{D}_{2} \mathrm{O}$, viewed along [001]. Displacement ellipsoids are displayed at the $90 \%$ probability level. For clarity, D atoms are not shown.

O6w) also serve as acceptor groups for one hydrogen bond. All other hydrogen bonds are directed towards the $\mathrm{O}$ atoms of the phosphate group, with $\mathrm{O} 1$ being twofold, $\mathrm{O} 2$ threefold, $\mathrm{O} 3$ fourfold and $\mathrm{O} 4$ threefold acceptor atoms, respectively (Fig. 3). Judging from the O..O distances [range 2.6931 (12)2.9025 (13) А; Table 2], hydrogen bonds of medium strength are formed in the crystal structure. The $\mathrm{PO}_{4}$ tetrahedron shows almost equal $\mathrm{P}-\mathrm{O}$ bond lengths typical of a fully deprotonated orthophosphate group (mean $1.546 \AA$ ), with marginal angular distortions.

A bond-valence analysis (Brown, 2002), using the parameters of Brese \& O'Keeffe (1991), reveals bond-valence sums (BVS, in valence units) of $\mathrm{K} 1=1.18, \mathrm{~K} 2=1.08, \mathrm{~K} 3=$

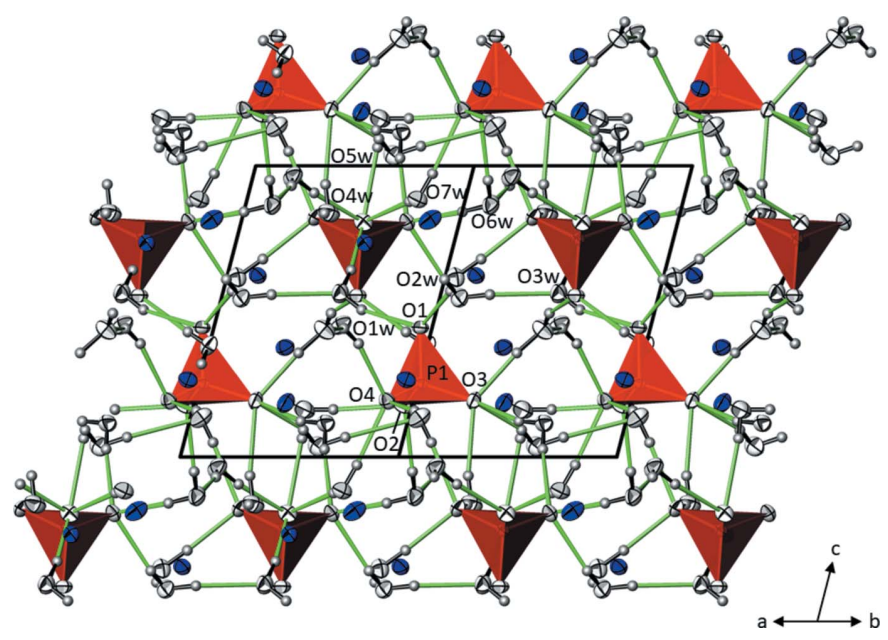

Figure 3

$\mathrm{O}-\mathrm{D} \cdots \mathrm{O}$ hydrogen-bonding network (green lines) in the crystal structure of $\mathrm{K}_{3} \mathrm{PO}_{4} \cdot 7 \mathrm{D}_{2} \mathrm{O}$, viewed along [101]. Displacement ellipsoids are displayed at the $90 \%$ probability level. 
1.11 , and $\mathrm{P} 1=4.85$, in good agreement with the expected values of +1 and +5 , respectively. The four oxygen atoms of the orthophosphate tetrahedron are considerably underbonded and show BVS values of $1.53(\mathrm{O} 1), 1.22(\mathrm{O} 2), 1.10$ $(\mathrm{O} 3)$ and $1.38(\mathrm{O} 4)$. O1 with the highest BVS of the four phosphate $\mathrm{O}$ atoms has two $\mathrm{K}^{+}$cations as additional bonding partners, $\mathrm{O} 4$ with the second highest BVS has one additional $\mathrm{K}^{+}$as bonding partner whereas $\mathrm{O} 2$ and $\mathrm{O} 3$ with the lowest $\mathrm{BVS}$ values are solely bonded to the $\mathrm{P}$ atom. The four $\mathrm{O}$ atoms compensate for underbonding by means of their role as acceptor atoms in hydrogen bonding (see above).

\section{Database survey}

In the Inorganic Structure Database (ICSD; Zagorac et al., 2019), the crystal structures of not less than 14 different phases in the system $\mathrm{K}_{2} \mathrm{O} / \mathrm{P}_{2} \mathrm{O}_{5} / \mathrm{H}_{2} \mathrm{O}$ are listed, including partly protonated $\mathrm{PO}_{4}$ or other condensed phosphate groups, and/or phases with water molecules. The only other phosphates of an alkali metal, thallium or ammonium with a fully deprotonated orthophosphate group are $\mathrm{Na}_{3} \mathrm{PO}_{4}\left(\mathrm{H}_{2} \mathrm{O}\right)_{8}$ (Larbot \& Durand, 1983), $\mathrm{Na}_{3} \mathrm{PO}_{4}\left(\mathrm{H}_{2} \mathrm{O}\right)_{0.5}$ (Averbuch-Pouchot \& Durif, 1983) and $\left(\mathrm{NH}_{4}\right)_{3}\left(\mathrm{PO}_{4}\right) \cdot 3 \mathrm{H}_{2} \mathrm{O}$ (Mootz \& Wunderlich, 1970). As a result of the different size of the $\mathrm{Na}^{+}$cation compared to $\mathrm{K}^{+}$, the role of $\mathrm{NH}_{4}{ }^{+}$as an active species in hydrogen bonding, and the different amounts of water molecules in these three crystal structures, there is no evident structural relation to $\mathrm{K}_{3} \mathrm{PO}_{4} \cdot 7 \mathrm{D}_{2} \mathrm{O}$.

\section{Synthesis and crystallization}

Commercial anhydrous $\mathrm{K}_{3} \mathrm{PO}_{4}$ (Sigma-Aldrich) was dissolved in a small amount of warm $\mathrm{D}_{2} \mathrm{O}$. Cooling to $255 \mathrm{~K}$ afforded rod-like crystals of the title heptahydrate that grew over night, with maximum edge lengths in the millimetre range.

\section{Refinement}

Crystal data, data collection and structure refinement details are summarized in Table 3. Positions of the D atoms were located in a difference-Fourier map and were refined freely under consideration of scattering factors for hydrogen atoms.

\section{References}

Averbuch-Pouchot, M. T. \& Durif, A. (1983). J. Solid State Chem. 46, 193-196.

Brese, N. E. \& O'Keeffe, M. (1991). Acta Cryst. B47, 192-197.

Brown, I. D. (2002). The Chemical Bond in Inorganic Chemistry: The Bond Valence Model. Oxford University Press.

Bruker (2016). SAINT-Plus, APEX2 and SADABS. Bruker AXS Inc., Madison, Wisconsin, USA.
Table 3

Experimental details.

\begin{tabular}{|c|c|}
\hline \multicolumn{2}{|l|}{ Crystal data } \\
\hline Chemical formula & $\mathrm{K}_{3} \mathrm{PO}_{4} \cdot 7 \mathrm{D}_{2} \mathrm{O}$ \\
\hline$M_{\mathrm{r}}$ & 352.5 \\
\hline Crystal system, space group & Monoclinic, $P 2_{1}$ \\
\hline Temperature $(\mathrm{K})$ & 100 \\
\hline$a, b, c(\AA)$ & $7.8325(7), 9.3406(8), 8.4471(7)$ \\
\hline$\beta\left(^{\circ}\right)$ & $108.727(2)$ \\
\hline$V\left(\AA^{3}\right)$ & $585.28(9)$ \\
\hline$Z$ & 2 \\
\hline Radiation type & Мо $K \alpha$ \\
\hline$\mu\left(\mathrm{mm}^{-1}\right)$ & 1.34 \\
\hline Crystal size $(\mathrm{mm})$ & $0.46 \times 0.09 \times 0.01$ \\
\hline \multicolumn{2}{|l|}{ Data collection } \\
\hline Diffractometer & Bruker Kappa APEXII CCD \\
\hline Absorption correction & $\begin{array}{l}\text { Multi-scan ( } S A D A B S \text {; Bruker, } \\
\text { 2016) }\end{array}$ \\
\hline$T_{\min }, T_{\max }$ & $0.54,0.99$ \\
\hline $\begin{array}{l}\text { No. of measured, independent and } \\
\text { observed }[I>3 \sigma(I)] \text { reflections }\end{array}$ & $9464,4273,4127$ \\
\hline$R_{\text {int }}$ & 0.021 \\
\hline$(\sin \theta / \lambda)_{\max }\left(\AA^{-1}\right)$ & 0.759 \\
\hline \multicolumn{2}{|l|}{ Refinement } \\
\hline$R\left[F^{2}>2 \sigma\left(F^{2}\right)\right], w R\left(F^{2}\right), S$ & $0.016,0.020,1.02$ \\
\hline No. of reflections & 4273 \\
\hline No. of parameters & 193 \\
\hline$\Delta \rho_{\max }, \Delta \rho_{\min }\left(\mathrm{e} \AA^{-3}\right)$ & $0.16,-0.13$ \\
\hline Absolute structure & $\begin{array}{l}2017 \text { Friedel pairs used in the } \\
\text { refinement (Flack, 1983) }\end{array}$ \\
\hline Absolute structure parameter & $0.004(16)$ \\
\hline
\end{tabular}

Computer programs: APEX2 and SAINT-Plus (Bruker, 2016), SHELXT (Sheldrick, 2015), JANA2006 (Petríčcek et al., 2014), ATOMS (Dowty, 2006) and publCIF (Westrip, 2010).

Dowty, E. (2006). ATOMS. Shape Software, Kingsport, Tennessee, USA.

Flack, H. D. (1983). Acta Cryst. A39, 876-881.

Gagné, O. C. \& Hawthorne, F. C. (2016). Acta Cryst. B72, 602 625.

Gmelin (1938). Gmelins Handbuch der Anorganischen Chemie, $8^{\text {th }}$ ed., Kalium 22, pp. 984-992. Berlin: Verlag Chemie.

Larbot, A. \& Durand, J. (1983). Acta Cryst. C39, 12-15.

Mootz, D. \& Wunderlich, H. (1970). Acta Cryst. B26, 1826-1835.

Petříček, V., Dušek, M. \& Palatinus, L. (2014). Z. Kristallogr. 229, 345-352.

Ring, J., Lindenthal, L., Weil, M. \& Stöger, B. (2017). Acta Cryst. E73, $1520-1522$.

Schroffenegger, M., Eder, F., Weil, M., Stöger, B., Schwendtner, K. \& Kolitsch, U. (2019). J. Alloys Compd, 820, 153369. https://doi.org/ 10.1016/j.jallcom.2019.153369.

Sheldrick, G. M. (2015). Acta Cryst. A71, 3-8.

Stöger, B. (2020). Unpublished results.

Stöger, B. \& Weil, M. (2014). Acta Cryst. C70, 7-11.

Stöger, B., Weil, M. \& Zobetz, E. (2012). Z. Kristallogr. 227, 859-868.

Westrip, S. P. (2010). J. Appl. Cryst. 43, 920-925.

Zagorac, D., Müller, H., Ruehl, S., Zagorac, J. \& Rehme, S. (2019). J. Appl. Cryst. 52, 918-925. 


\section{supporting information}

Acta Cryst. (2020). E76, 177-179 [https://doi.org/10.1107/S2056989020000201]

\section{Crystal structure of the deuterated heptahydrate of potassium phosphate, $\mathrm{K}_{3} \mathrm{PO}_{4} \cdot 7 \mathrm{D}_{2} \mathrm{O}$}

\section{Matthias Weil and Berthold Stöger}

Computing details

Data collection: APEX2 (Bruker, 2016); cell refinement: SAINT-Plus (Bruker, 2016); data reduction: SAINT-Plus (Bruker, 2016); program(s) used to solve structure: SHELXT (Sheldrick, 2015); program(s) used to refine structure: Jana2006 (Petř́íck et al., 2014); molecular graphics: ATOMS (Dowty, 2006); software used to prepare material for publication: publCIF (Westrip, 2010).

Potassium phosphate heptahydrate

Crystal data

$\mathrm{K}_{3} \mathrm{PO}_{4} \cdot 7 \mathrm{D}_{2} \mathrm{O}$

$M_{r}=352.5$

Monoclinic, $P 2_{1}$

Hall symbol: $\mathrm{P} 2 \mathrm{yb}$

$a=7.8325$ (7) $\AA$

$b=9.3406(8) \AA$

$c=8.4471(7) \AA$

$\beta=108.727(2)^{\circ}$

$V=585.28(9) \AA^{3}$

$Z=2$

$F(000)=348$

$D_{\mathrm{x}}=2.000 \mathrm{Mg} \mathrm{m}^{-3}$

Mo $K \alpha$ radiation, $\lambda=0.71073 \AA$

Cell parameters from 7382 reflections

$\theta=2.6-32.6^{\circ}$

$\mu=1.34 \mathrm{~mm}^{-1}$

$T=100 \mathrm{~K}$

Rod, colourless

$0.46 \times 0.09 \times 0.01 \mathrm{~mm}$

\section{Data collection}

Bruker Kappa APEXII CCD diffractometer

Radiation source: X-ray tube Graphite monochromator

$\omega$ - and $\varphi$-scans

Absorption correction: multi-scan

(SADABS; Bruker, 2016)

$T_{\min }=0.54, T_{\max }=0.99$

\section{Refinement}

Refinement on $F$

$R\left[F^{2}>2 \sigma\left(F^{2}\right)\right]=0.016$

$w R\left(F^{2}\right)=0.020$

$S=1.02$

4273 reflections

193 parameters

0 restraints

1 constraint
9464 measured reflections

4273 independent reflections

4127 reflections with $I>3 \sigma(I)$

$R_{\text {int }}=0.021$

$\theta_{\max }=32.6^{\circ}, \theta_{\min }=2.6^{\circ}$

$h=-10 \rightarrow 11$

$k=-14 \rightarrow 14$

$l=-12 \rightarrow 10$

Weighting scheme based on measured s.u.'s $w=$ $1 /\left(\sigma^{2}(F)+0.0001 F^{2}\right)$

$(\Delta / \sigma)_{\max }=0.019$

$\Delta \rho_{\max }=0.16 \mathrm{e} \AA^{-3}$

$\Delta \rho_{\min }=-0.13$ e $\AA^{-3}$

Absolute structure: 2017 Friedel pairs used in the refinement (Flack, 1983)

Absolute structure parameter: 0.004 (16) 
Fractional atomic coordinates and isotropic or equivalent isotropic displacement parameters $\left(\hat{A}^{2}\right)$

\begin{tabular}{|c|c|c|c|c|}
\hline & $x$ & $y$ & $z$ & $U_{\text {iso }} * / U_{\text {eq }}$ \\
\hline K1 & $0.42550(3)$ & $0.01804(2)$ & $0.73444(3)$ & $0.00950(6)$ \\
\hline $\mathrm{K} 2$ & $-0.14458(3)$ & $-0.00537(2)$ & $0.62341(3)$ & $0.00990(6)$ \\
\hline $\mathrm{K} 3$ & $0.52865(3)$ & $0.39152(2)$ & $0.81480(3)$ & $0.01224(6)$ \\
\hline P1 & $0.02505(3)$ & $0.04285(3)$ & $0.25261(3)$ & $0.00571(7)$ \\
\hline O1 & $0.08740(10)$ & $0.03291(8)$ & $0.44490(9)$ & 0.00823 (19) \\
\hline $\mathrm{O} 2$ & $-0.06671(10)$ & $-0.09902(8)$ & $0.17723(10)$ & $0.0101(2)$ \\
\hline $\mathrm{O} 3$ & $-0.10938(10)$ & $0.16905(8)$ & $0.19358(10)$ & $0.0092(2)$ \\
\hline $\mathrm{O} 4$ & $0.18924(10)$ & $0.07033(8)$ & $0.19270(10)$ & $0.0086(2)$ \\
\hline O1w & $0.50577(11)$ & $0.08478(8)$ & $0.45314(11)$ & $0.0119(2)$ \\
\hline $\mathrm{O} 2 \mathrm{w}$ & $0.22528(11)$ & $0.27301(8)$ & $0.61723(11)$ & $0.0106(2)$ \\
\hline $\mathrm{O} 3 \mathrm{w}$ & $0.23569(11)$ & $-0.22553(8)$ & $0.56243(11)$ & $0.0116(2)$ \\
\hline $\mathrm{O} 4 \mathrm{w}$ & $-0.18873(12)$ & $0.20109(9)$ & $0.83725(11)$ & $0.0129(2)$ \\
\hline $\mathrm{O} 5 \mathrm{w}$ & $0.53272(11)$ & $0.15028(9)$ & $1.03669(11)$ & $0.0126(2)$ \\
\hline O6w & $0.71314(11)$ & $-0.16355(8)$ & $0.86601(11)$ & $0.0119(2)$ \\
\hline $\mathrm{O} 7 \mathrm{w}$ & $0.17243(11)$ & $-0.04581(9)$ & $0.88427(10)$ & $0.0103(2)$ \\
\hline D11 & $0.564(2)$ & $0.161(2)$ & $0.442(2)$ & $0.027(5)^{*}$ \\
\hline D12 & $0.409(3)$ & $0.091(2)$ & $0.390(3)$ & $0.033(5)^{*}$ \\
\hline D21 & $0.172(3)$ & $0.315(2)$ & $0.680(2)$ & $0.034(5)^{*}$ \\
\hline D22 & $0.173(3)$ & $0.213(2)$ & $0.570(3)$ & $0.037(6)^{*}$ \\
\hline D31 & $0.182(2)$ & $-0.162(2)$ & $0.506(3)$ & $0.031(5)^{*}$ \\
\hline D32 & $0.179(3)$ & $-0.261(3)$ & $0.642(3)$ & $0.052(7)^{*}$ \\
\hline D41 & $-0.153(2)$ & 0.1885 (19) & $0.936(3)$ & $0.022(4) *$ \\
\hline D42 & $-0.113(3)$ & $0.258(3)$ & $0.837(3)$ & $0.049(7)^{*}$ \\
\hline D51 & $0.477(2)$ & 0.2098 (19) & $1.068(2)$ & $0.021(4)^{*}$ \\
\hline D52 & $0.634(2)$ & 0.1654 (19) & 1.093 (2) & $0.023(4)^{*}$ \\
\hline D61 & $0.776(3)$ & $-0.143(2)$ & $0.950(3)$ & $0.031(5)^{*}$ \\
\hline D62 & $0.759(2)$ & $-0.240(2)$ & $0.842(2)$ & $0.023(4)^{*}$ \\
\hline D71 & $0.132(2)$ & $-0.123(2)$ & $0.877(2)$ & $0.023(4)^{*}$ \\
\hline D72 & $0.166(2)$ & -0.0150 (19) & $0.973(2)$ & $0.019(4)^{*}$ \\
\hline
\end{tabular}

Atomic displacement parameters $\left(\AA^{2}\right)$

\begin{tabular}{lllllll}
\hline & $U^{11}$ & $U^{22}$ & $U^{33}$ & $U^{12}$ & $U^{13}$ & $U^{23}$ \\
\hline $\mathrm{K} 1$ & $0.01055(8)$ & $0.00951(9)$ & $0.00895(9)$ & $0.00141(6)$ & $0.00384(7)$ & $0.00041(7)$ \\
$\mathrm{K} 2$ & $0.00815(8)$ & $0.01323(9)$ & $0.00836(9)$ & $0.00028(7)$ & $0.00272(7)$ & $-0.00109(7)$ \\
$\mathrm{K} 3$ & $0.01205(9)$ & $0.00943(9)$ & $0.01200(11)$ & $-0.00261(7)$ & $-0.00067(8)$ & $0.00104(7)$ \\
P1 & $0.00639(10)$ & $0.00552(10)$ & $0.00530(11)$ & $-0.00037(8)$ & $0.00201(8)$ & $0.00003(8)$ \\
O1 & $0.0102(3)$ & $0.0084(3)$ & $0.0056(3)$ & $0.0002(2)$ & $0.0020(2)$ & $-0.0001(2)$ \\
O2 & $0.0131(3)$ & $0.0080(3)$ & $0.0086(3)$ & $-0.0040(3)$ & $0.0027(3)$ & $-0.0020(3)$ \\
O3 & $0.0086(3)$ & $0.0090(3)$ & $0.0099(4)$ & $0.0029(2)$ & $0.0028(3)$ & $0.0022(3)$ \\
O4 & $0.0089(3)$ & $0.0088(3)$ & $0.0092(4)$ & $-0.0002(2)$ & $0.0046(3)$ & $0.0004(2)$ \\
O1w & $0.0078(3)$ & $0.0150(4)$ & $0.0114(4)$ & $-0.0011(3)$ & $0.0010(3)$ & $0.0026(3)$ \\
O2w & $0.0091(3)$ & $0.0103(3)$ & $0.0120(4)$ & $-0.0011(3)$ & $0.0026(3)$ & $-0.0033(3)$ \\
O3w & $0.0127(3)$ & $0.0109(3)$ & $0.0123(4)$ & $0.0032(3)$ & $0.0056(3)$ & $0.0032(3)$ \\
O4w & $0.0182(4)$ & $0.0112(3)$ & $0.0102(4)$ & $-0.0007(3)$ & $0.0059(3)$ & $0.0003(3)$
\end{tabular}




\begin{tabular}{lllllll}
$\mathrm{O} 5 \mathrm{w}$ & $0.0084(3)$ & $0.0168(4)$ & $0.0122(4)$ & $-0.0016(3)$ & $0.0030(3)$ & $-0.0033(3)$ \\
$\mathrm{O} 6 \mathrm{w}$ & $0.0111(3)$ & $0.0099(3)$ & $0.0122(4)$ & $0.0020(3)$ & $0.0003(3)$ & $-0.0024(3)$ \\
$\mathrm{O} 7 \mathrm{w}$ & $0.0126(3)$ & $0.0098(3)$ & $0.0091(4)$ & $-0.0010(3)$ & $0.0041(3)$ & $-0.0008(3)$ \\
\hline
\end{tabular}

Geometric parameters $\left(\AA,{ }^{\circ}\right)$

\begin{tabular}{|c|c|c|c|}
\hline $\mathrm{K} 1-\mathrm{O} 5 \mathrm{w}$ & $2.7153(10)$ & $\mathrm{K} 3-\mathrm{O} 5 \mathrm{w}$ & $2.9246(10)$ \\
\hline $\mathrm{K} 1-\mathrm{O} 1 \mathrm{w}$ & $2.7183(11)$ & $\mathrm{P} 1-\mathrm{O} 1$ & $1.5414(8)$ \\
\hline $\mathrm{K} 1-\mathrm{O} 7 \mathrm{w}$ & $2.7381(10)$ & $\mathrm{P} 1-\mathrm{O} 2$ & $1.5440(8)$ \\
\hline $\mathrm{K} 1-\mathrm{O} 6 \mathrm{w}$ & $2.7532(9)$ & $\mathrm{P} 1-\mathrm{O} 4$ & $1.5472(10)$ \\
\hline $\mathrm{K} 1-\mathrm{O} 3 \mathrm{w}$ & $2.8479(9)$ & $\mathrm{P} 1-\mathrm{O} 3$ & $1.5523(8)$ \\
\hline $\mathrm{K} 1-\mathrm{O} 2 \mathrm{w}$ & $2.8486(9)$ & $\mathrm{O} 1 \mathrm{w}-\mathrm{D} 11$ & $0.86(2)$ \\
\hline $\mathrm{K} 1-\mathrm{O} 1$ & $2.9757(9)$ & $\mathrm{O} 1 \mathrm{w}-\mathrm{D} 12$ & $0.778(18)$ \\
\hline $\mathrm{K} 2-\mathrm{O} 1$ & $2.7317(10)$ & $\mathrm{O} 2 \mathrm{w}-\mathrm{D} 21$ & $0.87(2)$ \\
\hline $\mathrm{K} 2-\mathrm{O} 4 \mathrm{w}$ & $2.7391(10)$ & $\mathrm{O} 2 \mathrm{w}-\mathrm{D} 22$ & $0.73(2)$ \\
\hline $\mathrm{K} 2-\mathrm{O} 7 \mathrm{w}$ & $2.7659(9)$ & $\mathrm{O} 3 \mathrm{w}-\mathrm{D} 31$ & $0.796(19)$ \\
\hline $\mathrm{K} 2-\mathrm{O} 1 \mathrm{w}^{\mathrm{i}}$ & $2.7836(9)$ & $\mathrm{O} 3 \mathrm{w}-\mathrm{D} 32$ & $0.98(3)$ \\
\hline $\mathrm{K} 2-\mathrm{O} 2 \mathrm{w}^{\mathrm{ii}}$ & $2.8269(9)$ & $\mathrm{O} 4 \mathrm{w}-\mathrm{D} 41$ & $0.80(2)$ \\
\hline $\mathrm{K} 2-\mathrm{O} 3 \mathrm{w}^{\mathrm{iii}}$ & $3.0144(9)$ & $\mathrm{O} 4 \mathrm{w}-\mathrm{D} 42$ & $0.79(2)$ \\
\hline $\mathrm{K} 2-\mathrm{O} 6 \mathrm{w}^{\mathrm{i}}$ & $3.0151(10)$ & $\mathrm{O} 5 \mathrm{w}-\mathrm{D} 51$ & $0.80(2)$ \\
\hline $\mathrm{K} 3-\mathrm{O} 2 \mathrm{w}$ & $2.6665(9)$ & $\mathrm{O} 5 \mathrm{w}-\mathrm{D} 52$ & $0.795(17)$ \\
\hline $\mathrm{K} 3-\mathrm{O} 4^{\mathrm{iv}}$ & $2.7867(9)$ & O6w-D61 & $0.748(19)$ \\
\hline $\mathrm{K} 3-\mathrm{O} 4 \mathrm{w}^{\mathrm{v}}$ & $2.7983(10)$ & O6w-D62 & $0.85(2)$ \\
\hline $\mathrm{K} 3-\mathrm{O} 5 \mathrm{w}^{\mathrm{vi}}$ & $2.8344(10)$ & $\mathrm{O} 7 \mathrm{w}-\mathrm{D} 71$ & $0.783(19)$ \\
\hline $\mathrm{K} 3-\mathrm{O} 1 \mathrm{w}^{\mathrm{iv}}$ & $2.8394(10)$ & $\mathrm{O} 7 \mathrm{w}-\mathrm{D} 72$ & $0.823(19)$ \\
\hline $\mathrm{K} 3-\mathrm{O} 7 \mathrm{w}^{\mathrm{vi}}$ & $2.9094(9)$ & & \\
\hline $\mathrm{O} 1-\mathrm{K} 1-\mathrm{O} 1 \mathrm{w}$ & $70.45(2)$ & $\mathrm{O} 1 \mathrm{w}^{\mathrm{iv}}-\mathrm{K} 3-\mathrm{O} 5 \mathrm{w}^{\mathrm{vi}}$ & $79.89(3)$ \\
\hline $\mathrm{O} 1-\mathrm{K} 1-\mathrm{O} 2 \mathrm{w}$ & $55.25(2)$ & $\mathrm{O} 1 \mathrm{w}^{\mathrm{iv}}-\mathrm{K} 3-\mathrm{O} 7 \mathrm{w}^{\mathrm{vi}}$ & $114.35(2)$ \\
\hline $\mathrm{O} 1-\mathrm{K} 1-\mathrm{O} 3 \mathrm{w}$ & $55.73(2)$ & $\mathrm{O} 2 \mathrm{w}-\mathrm{K} 3-\mathrm{O} 4 \mathrm{w}^{\mathrm{v}}$ & $107.76(3)$ \\
\hline $\mathrm{O} 1-\mathrm{K} 1-\mathrm{O} 5 \mathrm{w}$ & $132.58(3)$ & $\mathrm{O} 2 \mathrm{w}-\mathrm{K} 3-\mathrm{O} 5 \mathrm{w}$ & $84.57(2)$ \\
\hline $\mathrm{O} 1-\mathrm{K} 1-\mathrm{O} 6 \mathrm{w}$ & $139.24(2)$ & $\mathrm{O} 2 \mathrm{w}-\mathrm{K} 3-\mathrm{O} 5 \mathrm{w}^{\mathrm{vi}}$ & $112.79(3)$ \\
\hline $\mathrm{O} 1-\mathrm{K} 1-\mathrm{O} 7 \mathrm{w}$ & $78.74(2)$ & $\mathrm{O} 2 \mathrm{w}-\mathrm{K} 3-\mathrm{O} 7 \mathrm{w}^{\mathrm{vi}}$ & $159.36(3)$ \\
\hline $\mathrm{O} 1 \mathrm{w}-\mathrm{K} 1-\mathrm{O} 2 \mathrm{w}$ & $76.14(3)$ & $\mathrm{O} 4 \mathrm{w}^{\mathrm{v}}-\mathrm{K} 3-\mathrm{O} 5 \mathrm{w}$ & $67.74(3)$ \\
\hline $\mathrm{O} 1 \mathrm{w}-\mathrm{K} 1-\mathrm{O} 3 \mathrm{w}$ & $88.09(3)$ & $\mathrm{O} 4 \mathrm{w}^{\mathrm{v}}-\mathrm{K} 3-\mathrm{O} 5 \mathrm{w}^{\mathrm{vi}}$ & $139.02(2)$ \\
\hline $\mathrm{O} 1 \mathrm{w}-\mathrm{K} 1-\mathrm{O} 5 \mathrm{w}$ & $128.98(3)$ & $\mathrm{O} 4 \mathrm{w}^{\mathrm{v}}-\mathrm{K} 3-\mathrm{O} 7 \mathrm{w}^{\mathrm{vi}}$ & $70.82(3)$ \\
\hline $\mathrm{O} 1 \mathrm{w}-\mathrm{K} 1-\mathrm{O} 6 \mathrm{w}$ & $96.04(3)$ & $\mathrm{O} 5 \mathrm{w}-\mathrm{K} 3-\mathrm{O} 5 \mathrm{w}^{\mathrm{vi}}$ & $109.99(3)$ \\
\hline $\mathrm{O} 1 \mathrm{w}-\mathrm{K} 1-\mathrm{O} 7 \mathrm{w}$ & $149.19(2)$ & $\mathrm{O} 5 \mathrm{w}-\mathrm{K} 3-\mathrm{O} 7 \mathrm{w}^{\mathrm{vi}}$ & $75.81(2)$ \\
\hline $\mathrm{O} 2 \mathrm{w}-\mathrm{K} 1-\mathrm{O} 3 \mathrm{w}$ & $110.59(2)$ & $\mathrm{O} 5 \mathrm{w}^{\mathrm{vi}}-\mathrm{K} 3-\mathrm{O} 7 \mathrm{w}^{\mathrm{vi}}$ & $69.21(2)$ \\
\hline $\mathrm{O} 2 \mathrm{w}-\mathrm{K} 1-\mathrm{O} 5 \mathrm{w}$ & $85.18(3)$ & $\mathrm{O} 1-\mathrm{P} 1-\mathrm{O} 2$ & $109.32(4)$ \\
\hline $\mathrm{O} 2 \mathrm{w}-\mathrm{K} 1-\mathrm{O} 6 \mathrm{w}$ & $160.60(2)$ & $\mathrm{O} 1-\mathrm{P} 1-\mathrm{O} 3$ & $109.69(5)$ \\
\hline $\mathrm{O} 2 \mathrm{w}-\mathrm{K} 1-\mathrm{O} 7 \mathrm{w}$ & $86.75(3)$ & $\mathrm{O} 1-\mathrm{P} 1-\mathrm{O} 4$ & $109.83(4)$ \\
\hline $\mathrm{O} 3 \mathrm{w}-\mathrm{K} 1-\mathrm{O} 5 \mathrm{w}$ & $142.78(3)$ & $\mathrm{O} 2-\mathrm{P} 1-\mathrm{O} 3$ & $109.96(4)$ \\
\hline $\mathrm{O} 3 \mathrm{w}-\mathrm{K} 1-\mathrm{O} 6 \mathrm{w}$ & $86.52(2)$ & $\mathrm{O} 2-\mathrm{P} 1-\mathrm{O} 4$ & $109.46(5)$ \\
\hline $\mathrm{O} 3 \mathrm{w}-\mathrm{K} 1-\mathrm{O} 7 \mathrm{w}$ & $74.04(3)$ & $\mathrm{O} 3-\mathrm{P} 1-\mathrm{O} 4$ & $108.56(4)$ \\
\hline $\mathrm{O} 5 \mathrm{w}-\mathrm{K} 1-\mathrm{O} 6 \mathrm{w}$ & $86.26(3)$ & $\mathrm{K} 2-\mathrm{O} 1-\mathrm{P} 1$ & $123.21(4)$ \\
\hline $\mathrm{O} 5 \mathrm{w}-\mathrm{K} 1-\mathrm{O} 7 \mathrm{w}$ & $73.48(3)$ & $\mathrm{K} 3^{\mathrm{vii}}-\mathrm{O} 4-\mathrm{P} 1$ & $131.03(4)$ \\
\hline $\mathrm{O} 6 \mathrm{w}-\mathrm{K} 1-\mathrm{O} 7 \mathrm{w}$ & $107.41(3)$ & $\mathrm{K} 1-\mathrm{O} 1 \mathrm{w}-\mathrm{K} 2^{\mathrm{v}}$ & $86.80(2)$ \\
\hline
\end{tabular}




\begin{tabular}{|c|c|}
\hline $\mathrm{O} 1-\mathrm{K} 2-\mathrm{O} 1 \mathrm{w}^{\mathrm{i}}$ & $113.18(3)$ \\
\hline $\mathrm{O} 1-\mathrm{K} 2-\mathrm{O} 2 \mathrm{w}^{\mathrm{ii}}$ & $74.56(3)$ \\
\hline $\mathrm{O} 1-\mathrm{K} 2-\mathrm{O} 3 \mathrm{w}^{\mathrm{iii}}$ & $71.77(3)$ \\
\hline $\mathrm{O} 1-\mathrm{K} 2-\mathrm{O} 4 \mathrm{w}$ & $121.27(3)$ \\
\hline $\mathrm{O} 1-\mathrm{K} 2-\mathrm{O} 6 \mathrm{w}^{\mathrm{i}}$ & $153.61(2)$ \\
\hline $\mathrm{O} 1-\mathrm{K} 2-\mathrm{O} 7 \mathrm{w}$ & $82.62(3)$ \\
\hline $\mathrm{O} 1 \mathrm{w}^{\mathrm{i}}-\mathrm{K} 2-\mathrm{O} 2 \mathrm{w}^{\mathrm{ii}}$ & $83.93(2)$ \\
\hline $\mathrm{O} 1 \mathrm{w}^{\mathrm{i}}-\mathrm{K} 2-\mathrm{O} 3 \mathrm{w}^{\mathrm{iii}}$ & $55.91(2)$ \\
\hline $\mathrm{O} 1 \mathrm{w}^{\mathrm{i}}-\mathrm{K} 2-\mathrm{O} 4 \mathrm{w}$ & $79.41(3)$ \\
\hline $\mathrm{O} 1 \mathrm{w}^{\mathrm{i}}-\mathrm{K} 2-\mathrm{O} 6 \mathrm{w}^{\mathrm{i}}$ & $88.99(3)$ \\
\hline $\mathrm{O} 1 \mathrm{w}^{\mathrm{i}}-\mathrm{K} 2-\mathrm{O} 7 \mathrm{w}$ & $159.32(3)$ \\
\hline $\mathrm{O} 2 \mathrm{w}^{\mathrm{ii}}-\mathrm{K} 2-\mathrm{O} 3 \mathrm{w}^{\mathrm{iii}}$ & $107.43(3)$ \\
\hline $\mathrm{O} 2 \mathrm{w}^{\mathrm{ii}}-\mathrm{K} 2-\mathrm{O} 4 \mathrm{w}$ & $160.62(3)$ \\
\hline $\mathrm{O} 2 \mathrm{w}^{\mathrm{ii}}-\mathrm{K} 2-\mathrm{O} 6 \mathrm{w}^{\mathrm{i}}$ & $94.81(3)$ \\
\hline $\mathrm{O} 2 \mathrm{w}^{\mathrm{ii}}-\mathrm{K} 2-\mathrm{O} 7 \mathrm{w}$ & $114.15(3)$ \\
\hline $\mathrm{O} 3 \mathrm{w}^{\mathrm{iii}}-\mathrm{K} 2-\mathrm{O} 4 \mathrm{w}$ & $70.97(3)$ \\
\hline $\mathrm{O} 3 \mathrm{w}^{\mathrm{iii}}-\mathrm{K} 2-\mathrm{O} 6 \mathrm{w}^{\mathrm{i}}$ & $134.55(3)$ \\
\hline $\mathrm{O} 3 \mathrm{w}^{\mathrm{iii}}-\mathrm{K} 2-\mathrm{O} 7 \mathrm{w}$ & $122.36(2)$ \\
\hline $\mathrm{O} 4 \mathrm{w}-\mathrm{K} 2-\mathrm{O} 6 \mathrm{w}^{\mathrm{i}}$ & $75.19(3)$ \\
\hline $\mathrm{O} 4 \mathrm{w}-\mathrm{K} 2-\mathrm{O} 7 \mathrm{w}$ & $80.90(3)$ \\
\hline $\mathrm{O} 6 \mathrm{w}^{\mathrm{i}}-\mathrm{K} 2-\mathrm{O} 7 \mathrm{w}$ & $79.91(3)$ \\
\hline $\mathrm{O} 4^{\mathrm{iv}}-\mathrm{K} 3-\mathrm{O} 1 \mathrm{w}^{\mathrm{iv}}$ & $58.26(2)$ \\
\hline $\mathrm{O} 4^{\mathrm{iv}}-\mathrm{K} 3-\mathrm{O} 2 \mathrm{w}$ & $142.03(3)$ \\
\hline $\mathrm{O} 4^{\mathrm{iv}}-\mathrm{K} 3-\mathrm{O} 4 \mathrm{w}^{\mathrm{v}}$ & $76.51(3)$ \\
\hline $\mathrm{O} 4^{\mathrm{iv}}-\mathrm{K} 3-\mathrm{O} 5 \mathrm{w}$ & $129.11(2)$ \\
\hline $\mathrm{O} 4^{\mathrm{iv}}-\mathrm{K} 3-\mathrm{O} 5 \mathrm{w}^{\mathrm{vi}}$ & $75.37(3)$ \\
\hline $\mathrm{O} 4^{\mathrm{iv}}-\mathrm{K} 3-\mathrm{O} 7 \mathrm{w}^{\mathrm{vi}}$ & $58.52(2)$ \\
\hline $\mathrm{O} 1 \mathrm{w}^{\mathrm{iv}}-\mathrm{K} 3-\mathrm{O} 2 \mathrm{w}$ & $85.85(3)$ \\
\hline $\mathrm{O} 1 \mathrm{w}^{\mathrm{iv}}-\mathrm{K} 3-\mathrm{O} 4 \mathrm{w}^{\mathrm{v}}$ & $109.19(3)$ \\
\hline $\mathrm{O} 1 \mathrm{w}^{\mathrm{iv}}-\mathrm{K} 3-\mathrm{O} 5 \mathrm{w}$ & $168.35(2)$ \\
\hline
\end{tabular}

\begin{tabular}{|c|c|}
\hline $\mathrm{K} 1-\mathrm{O} 1 \mathrm{w}-\mathrm{K} 3^{\mathrm{vii}}$ & $124.16(3)$ \\
\hline $\mathrm{K} 2^{\mathrm{v}}-\mathrm{O} 1 \mathrm{w}-\mathrm{K} 3^{\mathrm{vii}}$ & $92.60(3)$ \\
\hline $\mathrm{K} 1-\mathrm{O} 2 \mathrm{w}-\mathrm{K} 2^{\mathrm{iii}}$ & $146.43(4)$ \\
\hline $\mathrm{K} 1-\mathrm{O} 2 \mathrm{w}-\mathrm{K} 3$ & $81.32(2)$ \\
\hline 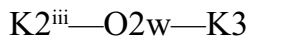 & $95.43(3)$ \\
\hline $\mathrm{D} 21-\mathrm{O} 2 \mathrm{w}-\mathrm{D} 22$ & $113(3)$ \\
\hline $\mathrm{K} 1-\mathrm{O} 3 \mathrm{w}-\mathrm{D} 31$ & $78.3(13)$ \\
\hline $\mathrm{K} 1-\mathrm{O} 3 \mathrm{w}-\mathrm{D} 32$ & $101.6(14)$ \\
\hline $\mathrm{D} 31-\mathrm{O} 3 \mathrm{w}-\mathrm{D} 32$ & $114(2)$ \\
\hline $\mathrm{K} 2-\mathrm{O} 4 \mathrm{w}-\mathrm{K} 3^{\mathrm{i}}$ & $131.87(3)$ \\
\hline $\mathrm{K} 2-\mathrm{O} 4 \mathrm{w}-\mathrm{D} 41$ & $120.6(14)$ \\
\hline $\mathrm{K} 2-\mathrm{O} 4 \mathrm{w}-\mathrm{D} 42$ & $102.2(19)$ \\
\hline $\mathrm{K} 33^{\mathrm{i}}-\mathrm{O} 4 \mathrm{w}-\mathrm{D} 41$ & $99.7(15)$ \\
\hline $\mathrm{K} 3 \mathrm{i}^{\mathrm{i}}-\mathrm{O} 4 \mathrm{w}-\mathrm{D} 42$ & $98.8(17)$ \\
\hline $\mathrm{D} 41-\mathrm{O} 4 \mathrm{w}-\mathrm{D} 42$ & $95(2)$ \\
\hline $\mathrm{K} 1-\mathrm{O} 5 \mathrm{w}-\mathrm{K} 3^{\text {viii }}$ & $89.03(2)$ \\
\hline $\mathrm{K} 1-\mathrm{O} 5 \mathrm{w}-\mathrm{D} 51$ & $126.3(11)$ \\
\hline $\mathrm{K} 1-\mathrm{O} 5 \mathrm{w}-\mathrm{D} 52$ & $126.4(16)$ \\
\hline $\mathrm{K} 3^{\text {viii }}-\mathrm{O} 5 \mathrm{w}-\mathrm{D} 51$ & $104.9(14)$ \\
\hline $\mathrm{K} 3^{\mathrm{viii}}-\mathrm{O} 5 \mathrm{w}-\mathrm{D} 52$ & $99.4(13)$ \\
\hline D51-O5w-D52 & $102.5(18)$ \\
\hline $\mathrm{K} 1-\mathrm{O} 6 \mathrm{w}-\mathrm{D} 61$ & $115.4(16)$ \\
\hline $\mathrm{K} 1-\mathrm{O} 6 \mathrm{w}-\mathrm{D} 62$ & $140.5(11)$ \\
\hline D61-O6w-D62 & $104(2)$ \\
\hline $\mathrm{K} 1-\mathrm{O} 7 \mathrm{w}-\mathrm{K} 2$ & $101.53(3)$ \\
\hline $\mathrm{K} 1-\mathrm{O} 7 \mathrm{w}-\mathrm{D} 71$ & $120.0(16)$ \\
\hline $\mathrm{K} 1-\mathrm{O} 7 \mathrm{w}-\mathrm{D} 72$ & $127.9(12)$ \\
\hline $\mathrm{K} 2-\mathrm{O} 7 \mathrm{w}-\mathrm{D} 71$ & $80.0(11)$ \\
\hline $\mathrm{K} 2-\mathrm{O} 7 \mathrm{w}-\mathrm{D} 72$ & $112.2(11)$ \\
\hline $\mathrm{D} 71-\mathrm{O} 7 \mathrm{w}-\mathrm{D} 72$ & $105(2)$ \\
\hline
\end{tabular}

Symmetry codes: (i) $x-1, y, z$; (ii) $-x, y-1 / 2,-z+1$; (iii) $-x, y+1 / 2,-z+1$; (iv) $-x+1, y+1 / 2,-z+1$; (v) $x+1, y, z$; (vi) $-x+1, y+1 / 2,-z+2$; (vii) $-x+1, y-1 / 2$, $-z+1$; (viii) $-x+1, y-1 / 2,-z+2$.

Hydrogen-bond geometry $\left(\AA,{ }^{\circ}\right)$

\begin{tabular}{lllll}
\hline$D-\mathrm{H} \cdots A$ & $D-\mathrm{H}$ & $\mathrm{H} \cdots A$ & $D \cdots A$ & $D-\mathrm{H} \cdots A$ \\
\hline $\mathrm{O} 1 w-\mathrm{D} 11 \cdots \mathrm{O} 3 w^{\text {iv }}$ & $0.86(2)$ & $1.91(2)$ & $2.7255(13)$ & $158(2)$ \\
$\mathrm{O} 1 w-\mathrm{D} 12 \cdots \mathrm{O} 4$ & $0.78(2)$ & $1.98(2)$ & $2.7391(11)$ & $164(2)$ \\
$\mathrm{O} 2 w-\mathrm{D} 21 \cdots \mathrm{O} 2^{\mathrm{iii}}$ & $0.87(2)$ & $1.85(2)$ & $2.7149(13)$ & $177(2)$ \\
$\mathrm{O} 2 w-\mathrm{D} 22 \cdots \mathrm{O} 1$ & $0.73(2)$ & $1.99(2)$ & $2.7029(11)$ & $167(3)$ \\
$\mathrm{O} 3 w-\mathrm{D} 31 \cdots \mathrm{O} 1$ & $0.80(2)$ & $1.97(2)$ & $2.7242(11)$ & $159(2)$ \\
$\mathrm{O} 3 w-\mathrm{D} 32 \cdots \mathrm{O} 3^{\mathrm{ii}}$ & $0.98(3)$ & $1.77(3)$ & $2.7395(14)$ & $171(2)$ \\
$\mathrm{O} 4 w-\mathrm{D} 41 \cdots \mathrm{O} 3^{\mathrm{ix}}$ & $0.80(2)$ & $2.10(2)$ & $2.8870(14)$ & $169(2)$ \\
$\mathrm{O} 4 w-\mathrm{D} 42 \cdots \mathrm{O} 2^{\mathrm{iii}}$ & $0.79(2)$ & $1.97(2)$ & $2.7679(13)$ & $177(2)$ \\
$\mathrm{O} 5 w-\mathrm{D} 51 \cdots \mathrm{O} 6 w^{\mathrm{vi}}$ & $0.80(2)$ & $2.11(2)$ & $2.9025(13)$ & $168(2)$ \\
$\mathrm{O} 5 w-\mathrm{D} 52 \cdots \mathrm{O} 3^{\mathrm{x}}$ & $0.80(2)$ & $1.92(2)$ & $2.6944(12)$ & $166(2)$ \\
$\mathrm{O} 6 w-\mathrm{D} 61 \cdots \mathrm{O} 2^{\mathrm{x}}$ & $0.75(2)$ & $1.96(2)$ & $2.7087(12)$ & $176(2)$ \\
$\mathrm{O} 6 w-\mathrm{D} 62 \cdots \mathrm{O} 44^{\mathrm{vii}}$ & $0.85(2)$ & $1.86(2)$ & $2.6931(12)$ & $165(2)$
\end{tabular}


supporting information

$\begin{array}{llllr}\mathrm{O} 7 w-\mathrm{D} 71 \cdots \mathrm{O} 3^{\mathrm{ii}} & 0.78(2) & 2.02(2) & 2.7498(12) & 155(2) \\ \mathrm{O} 7 w-\mathrm{D} 72 \cdots{ }^{\text {ix }} & 0.82(2) & 1.97(2) & 2.7859(13) & 171(2)\end{array}$

Symmetry codes: (ii) $-x, y-1 / 2,-z+1$; (iii) $-x, y+1 / 2,-z+1$; (iv) $-x+1, y+1 / 2,-z+1$; (vi) $-x+1, y+1 / 2,-z+2$; (vii) $-x+1, y-1 / 2,-z+1$; (ix) $x, y, z+1$; (x) $x+1$, $y, z+1$. 